\title{
Multilevel solution of the elastohydrodynamically lubricated circular contact problem Part 2: smooth surface results
}

\author{
C. H. Venner and W. E. ten Napel \\ Faculty of Mechanical Engineering, University of Twente, PO Box 217, 7500AE Enschede \\ (Netherlands)
}

(Received May 1, 1991; accepted July 29, 1991)

\begin{abstract}
A fast multilevel solver for the solution of the elastohydrodynamically lubricated circular contact problem has been applied to the standard situation assuming the running surfaces to be perfectly smooth. Pressure profiles and film shapes for various operating conditions, are shown and overview graphs of the minimum, central and average film thickness as a function of the governing parameters covering a wide range of operating conditions are presented.
\end{abstract}

\section{Introduction}

The first part of this paper outlines the development of a fast solver for elastohydrodynamically lubricated (EHL) point contact problems. This second part presents the results obtained with this solver for the circular contact situation assuming the surfaces to be perfectly smooth.

First the variation of the pressure profiles and the associated film shapes with the governing parameters is shown. Subsequently, the dependence of some characteristic film thicknesses on the operating conditions is investigated and, in particular, overview graphs are presented giving the minimum, central and average film thickness as a function of the governing parameters. From the calculational results a formula has been derived that accurately predicts the central film thickness in the entire parameter range considered.

The results presented in Part I of this study showed that the algorithm allows solution of the problem using a large nodal density. The results presented here demonstrate that the algorithm also meets a second essential requirement for the study of more complex (non-smooth) EHL circular contact situations, i.e. it is very stable. Results of the application of the algorithm to circular contacts with various types of surface features can be found in ref. 1 .

\section{Solutions}

First the variation of the pressure profile and film shape with variation of the operating conditions is investigated. The results are presented in terms of the Moes 
dimensionless point contact parameters, i.e. a load parameter $M$ and a materials parameter $L$ (see nomenclature). However, for reasons of comparison also values of alternative dimensionless parameters, eg. the Hamrock and Dowson dimensionless parameters, $W, G$, and $U$, characterizing the operating conditions are given. The Moes dimensionless parameters are related to the latter parameters according to

$M=W(2 U)^{-3 / 4}$

$L=G(2 U)^{1 / 4}$

\subsection{Calculational details}

The majority of the presented solutions have been calculated on a domain $\{(X$, $\left.Y) \in \mathbb{R}^{2} \mid-4.5 \leq X \leq 1.5,-3 \leq Y \leq 3\right\}$. However, in very lightly loaded situations a larger calculational domain has been used. For these conditions the use of a large domain is essential. If the domain, and particularly the inlet region, is not chosen large enough it results in an underestimation of the film thickness. This numerical artefact is referred to as numerical starvation, see e.g. refs. 2,3 and also ref. 1 . In heavily loaded situations a smaller calculational domain than mentioned above is sufficient to obtain an accurate estimate of the film shape, eg. the choice of $X_{a}=-2.5, X_{b}=1.5$ and $Y_{a}=2$ serves well.

All results have been obtained assuming the lubricant to be compressible according to the relation proposed by Dowson and Higginson [5] and using the Roelands [4] viscosity pressure equation.

Because the surfaces are assumed to be perfectly smooth, the solutions for the pressure and the associated film shapes must be symmetric with respect to the centerline of the contact, i.e. $Y=0$. Until now, similar point contact calculations have been performed using this symmetry condition as an explicit boundary condition. This enables solution of the problem on only half of the domain, see eg. ref. 2. For two reasons this approach has not been followed here. Firstly, using the full domain is much more convenient in the calculation of the elastic deformation integrals with the multilevel multi-integration algorithm, see ref. 1 . Secondly, and even more important, it would limit future studies of the effect of surface features to symmetric features only. Hence, the problem is solved on the entire domain with only the usual Dirichlet condition $P=0$ on all four boundaries of the calculational domain. Consequently, in the present application (calculational domain and undeformed gap symmetric with respect to $Y$ ), the symmetry in the solutions should appear naturally.

\subsection{Varying $M$ and $L$}

Figure 1(a) presents the pressure profile for a relatively lightly loaded situation, i.e. $M=10$, and $L=10$. This corresponds with the following values of the Hamrock and Dowson parameters: $W=9.46 \times 10^{-8}, G=4729$, and $U=1.0 \times 10^{-11}$. The values of the parameters $\bar{\alpha}$ and $\lambda$ are: $\bar{\alpha}=7.85, \lambda=0.51$. Hence, for $\alpha=1.7 \times 10^{-8}$ the maximum Hertzian pressure for this load situation is only $0.46 \mathrm{GPa}$. The solution has been calculated using 263169 nodes on the entire domain which extended from $X_{a}=-9$ to $X_{b}=3$ in the $X$ direction and from $-Y_{a}$ to $Y_{a}$ in the $Y$ direction with $Y_{a}=6$. This large domain was used to avoid numerical starvation. The associated film shape is shown in Fig. 1(b). Note the reversion of the vertical axis.

In spite of the relatively small load the pressure profile and film shape show the characteristic EHL features. The pressure profile more or less approximates a semiellipsoid and because of the low load in the inlet region the pressure smoothly builds up to this semi-ellipsoid. Furthermore, the pressurc shows the three-dimensional equivalent of the pressure spike just ahead of the cavitated region. Obviously the local 


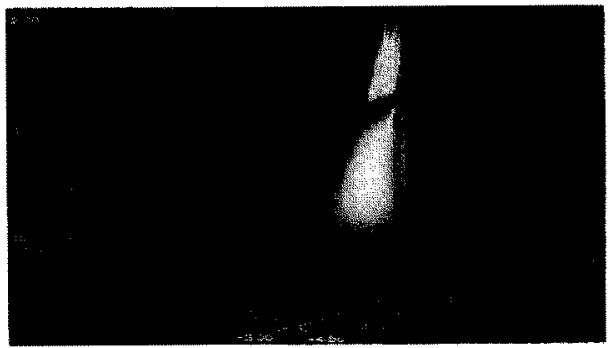

(a)

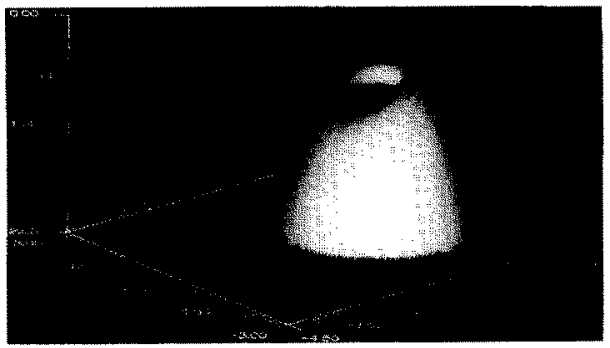

(b)

Fig. 1. (a) Pressure profile $M=10$ and $L=10$ ( $W=9.46 \times 10^{-8}, U=1.0 \times 10^{-11}, G=4729$, or $\bar{\alpha}=7.85$ and $\lambda=0.51$ ). (b) Film shape associated with the pressure profile presented in (a), i.e. $M=10$ and $L=10$.

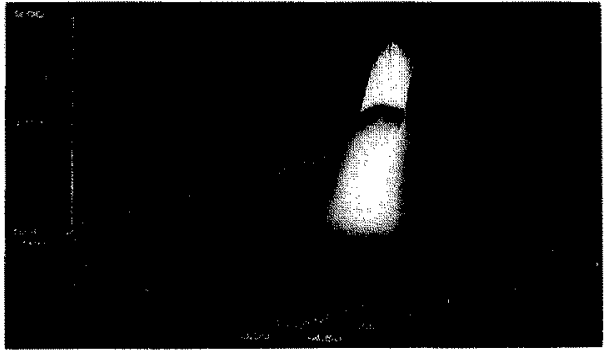

(a)

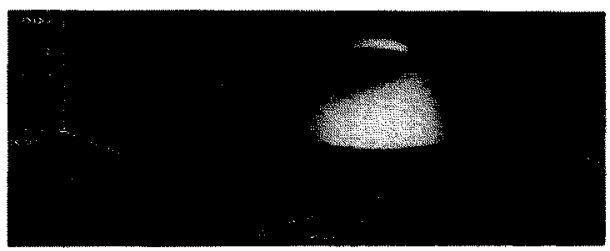

(b)

Fig. 2. (a) Pressure profile $M=20$ and $L=10\left(W=1.89 \times 10^{-7}, U=1.0 \times 10^{-11}, G=4729\right.$, or $\bar{\alpha}=9.89$ and $\lambda=0.2$ ). (b) Film shape associated with the pressure profile presented in (a), i.e. $M=20$ and $L=10$.

nodal density in the spike region is not large enough to smoothly describe the pressure profile in the spike region and consequently it looks ragged. The film shape shows the well known horseshoe-shaped region where in this particular low-load situation the side lobes where the minimum film thickness occurs are relatively large. This can be seen from Fig. 1(b) but is more obvious from a contour plot of the film thickness, e.g. see Fig. 7.

To demonstrate the effect of load, the pressure profiles and the associated film shapes for varying $M$ and constant $L(L=10)$ are displayed in Figs. 2-4. In addition to this variation of $M$, Figs. 5 and 6 give the pressure profile and the associated film shapes for $M=200$ and $L=0$ and $L=5$ respectively. Together with Fig. 3 the latter figures display the characteristic changes in the solutions with increasing dependence of the viscosity on the pressure. Finally Fig. 7 gives the film thickness contour plots for a number of load conditions among which the situations presented in Figs. 1-6.

The variations in the solutions with increasing $M$ for a given $L$ resemble the tendencies discussed for the line contact problem in ref. 1. With increasing $M$ (load) the pressure profile approximates the Hertzian dry contact pressure and both the region of pressure generation (inlet) and the pressure spike narrow down. In addition, as for the line contact problem, the minimum film thickness decreases with increasing $M$. 


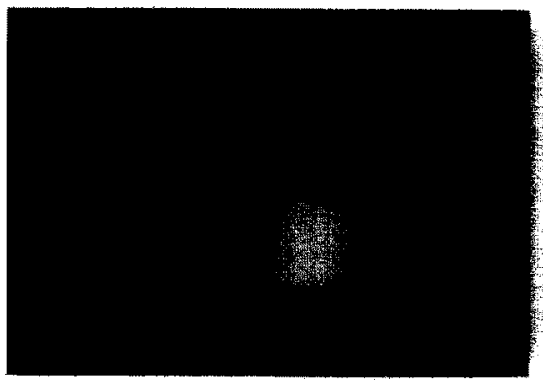

(a)

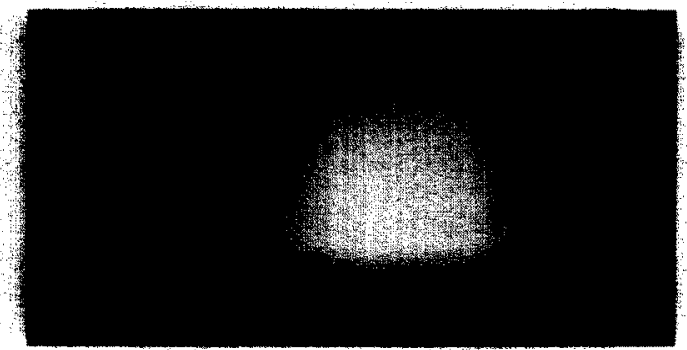

(b)

Fig. 3. (a) Pressure profile $M=200$ and $L=10\left(W=1.89 \times 10^{-6}, U=1.0 \times 10^{-14}, G=479\right)$ or $\tilde{\alpha}=21.31$ and $\lambda=9.39 \times 10^{-3}$ ). (b) Film shape associated with the pressure profile presented in (a), i.e. $M=200$ and $L=10$.

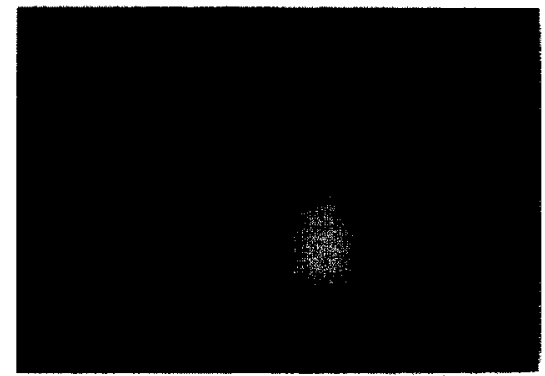

(a)

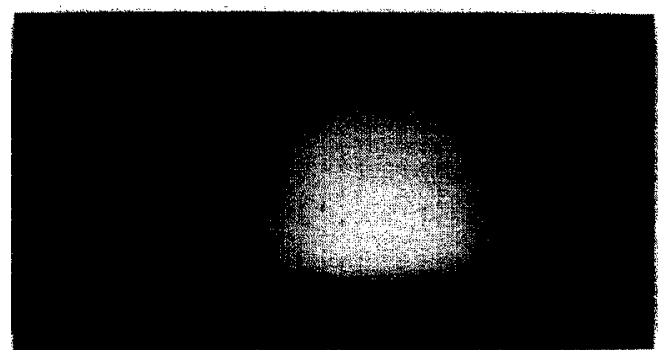

(b)

Fig. 4. (a) Pressure profile $M=1000$ and $L=10\left(W=9.46 \times 10^{-6}, U=1.0 \times 10^{-11}, G=4729, \mathrm{or}\right.$ $\bar{\alpha}=36.44$ and $\lambda=1.10 \times 10^{-3}$ ). (b) Film shape associated with the pressure profile presented in (a), i.e. $M=1000$ and $L=10$.

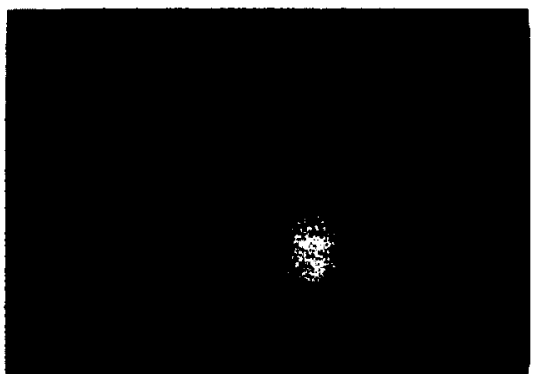

(a)

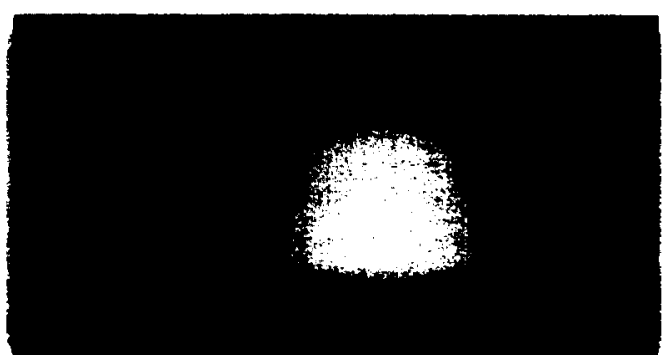

(b)

Fig. 5. (a) Pressure profile $M=200$ and $L=0\left(W=1.89 \times 10^{-6}, U=1.0 \times 10^{-11}, G=0\right.$, or $\bar{\alpha}=0$ and $\lambda=9.39 \times 10^{-3}$ ). (b) Film shape associated with the pressure profile presented in (a), i.e. $M=200$ and $L=0$.

Characteristic for the circular contact situation is the formation of the horseshoeshaped region in the film thickness, i.e. the sidelobes. These sidelobes can be recognized clearly in the film thickness plots and it can be seen that with increasing $M$ they 


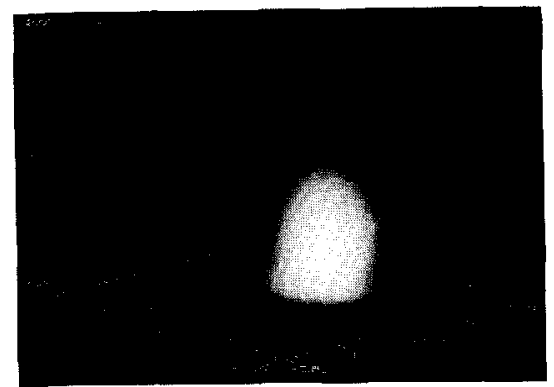

(a)

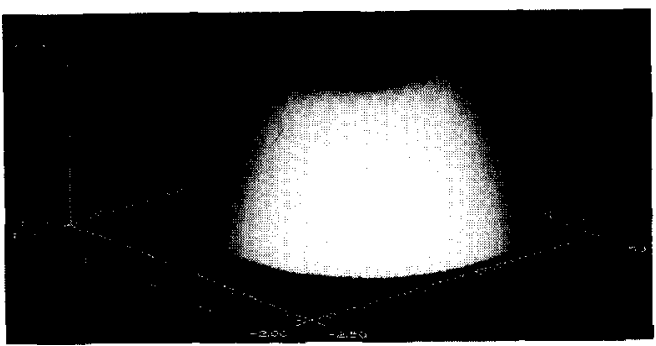

(b)

Fig. 6. (a) Pressure profile $M=200$ and $L=5\left(W=1.89 \times 10^{-6}, U=1.0 \times 10^{-11}, G=2364\right.$, or $\bar{\alpha}=10.65$ and $\lambda=9.39 \times 10^{-3}$ ). (b) Film shape associated with the pressure profile presented in (a), i.e. $M=200$ and $L=5$.
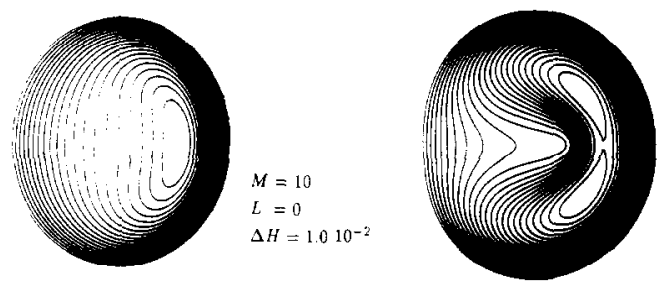

$M=10$

$\Delta H=1010^{-2}$
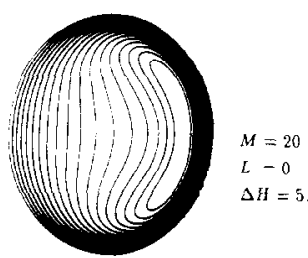

$L-0$

$\Delta H=5.010^{-3}$
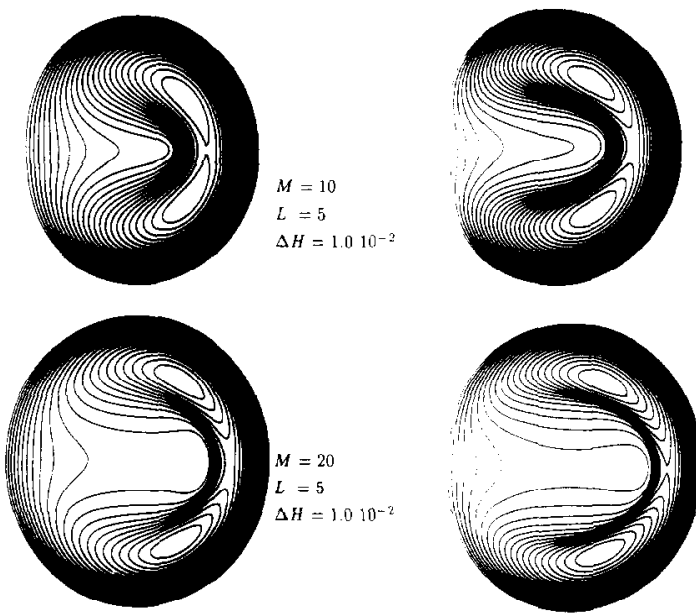

$M=i$

$L=10$

$3 H=\left[.030^{-2}\right.$
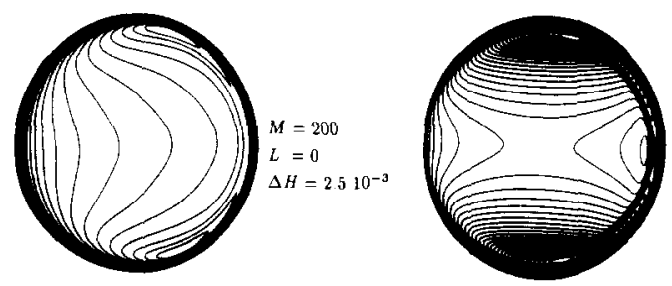

$M=200$

$L=5$

$\Delta H=1.2510^{-3}$

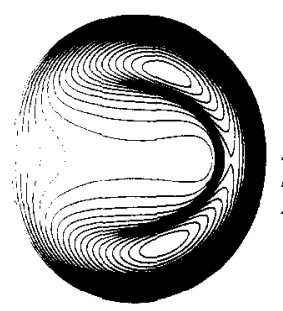

$M=2 \mid$

L 11

$\Delta n=1010^{-2}$

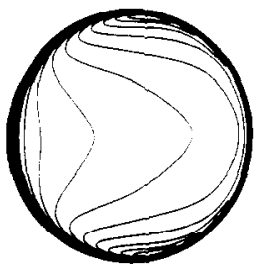

$M=1000$

$L=0$

$\Delta H=5.010^{-4}$

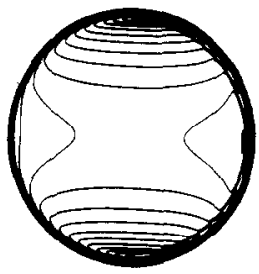

$M=1000$

$L=5$

$\Delta H=1.2510^{-3}$

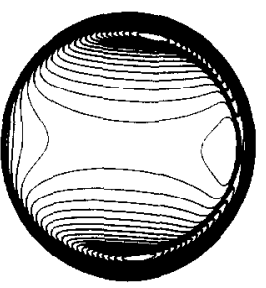

$M=200$

$L=10$

$\Delta H=2.510^{-3}$

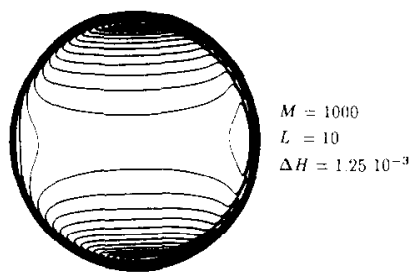

Fig. 7. Film thickness contour plots for various load conditions. 
become smaller and "move" in the outward direction until for large $M$ the minimum film thickness is practically found at the side of the contact, i.e. at $X=0, Y= \pm 1$. This can be seen from the photographs but is even more obvious from the film thickness contour plots of Fig. 7. This decrease in size of the sidelobes forms a complication with respect to the accurate estimation of the minimum film thickness. With increasing $M$ this becomes more difficult, as was also observed by Kweh et al. [3] and will be discussed in more detail in Section 3.2.

Comparison of the pressure profiles presented in Figs, 5(a), 6(a) and 2(a) shows the influence of the fact that the viscosity increases with pressure on the solution. Firstly, the pressure profile for the isoviscous situation $(L=0)$ presented in Fig. 5(a) clearly has no spike and for this relatively high value of $M$ it closely approximates the Hertzian semi-ellipsoid pressure profile. With increasing $L$ a pressure spike gradually develops in an analogous way as was found for the line contact problem (see ref. 6 ). This is illustrated by Fig. 6(a) $(L=5)$ where "the spike" shows up in the form of a ridge that can still be described accurately with the present nodal density $\left(\left(n_{x}+1\right) \times\left(n_{y}+1\right)=263169\right)$. Subsequently, with increasing $L$. its height increases and it becomes a real "spike", see e.g. Fig. 2(a) where it is fully developed. In that siluationt the local nodal density of the applied grid is obviously not large enough to lescribe the spike accurately and consequently it looks ragged.

Another effect related to the increasing pressure dependence of the viscosity can be seen from the film thickness contour plots of Fig. 7 . For a given value of $L$ with increasing $M$ the value of $\ddot{\alpha}$ and in fact the maximum Hertzian pressure increases. Consequently, the viscosity is higher and the flow in the contact region gradually approximates a pure shear flow. This follows from the fact that the film thickness contour plots become almost symmetric with respect to the $Y$-axis. This effect is particularly visible in the contour plot for $M=1000$ and $L=10$. With $\alpha=1,7 \times 10$ the latter solution represents a maximum Hertzian pressure of $2.14 \mathrm{GPa}$.

\section{Film thickness}

For the line contact problem the natural choice for a film thickness parameter to be used in overview graphs was the minimum film thickness. In the case of point contact problems the situation is more complex. Because of the additional dimension the fluid can flow around a region of small film thickness and, as a result, the ratio between the central film thickness (the film thickness at the location where hoth $a P$ / $\partial X=0$ and $\partial P / \partial Y=0$ ) and the minimum film thickness is not necessarily a constant. This has indeed been observed over the past few years (see e.g. refs. 2 and 3), and the value of this ratio, $H_{\mathrm{c}} / H_{\mathrm{m}}$, as a function of $M$ and $L$ obtained from the present calculations is displayed in Table 1.

This table clearly shows that the ratio is not a constant and consequently the minimum and central film thickness differ in their dependence on the load conditions. Hence the question arises which parameter can be used best to present an overview of the results: the minimum film thickness, the central film thickness or maybe an average film thickness. In this work graphs of all threc will be presented. Before doing so, Section 3.1 discusses some asymptotic solutions for the central and the minimum film thickness that have been presented so far. Subsequently in Section 3.2 the film thickness diagrams are given and in Section 3.3 a formula is presented predicting the central film thickness as a function of the operating conditions. 
TABLE 1

Ratio $H_{c} / H_{\mathrm{m}}$ as a function of $M$ and $L$

\begin{tabular}{cccccccccc}
\hline$L \backslash M$ & 3 & 5 & 10 & 20 & 50 & 100 & 200 & 500 & 1000 \\
\hline 0 & 1.3 & 1.3 & 1.3 & 1.3 & 1.4 & 1.5 & 1.6 & 1.7 & 1.9 \\
1 & 1.3 & 1.3 & 1.3 & 1.4 & 1.5 & 1.7 & 1.9 & 2.3 & 2.7 \\
2.5 & 1.3 & 1.3 & 1.3 & 1.5 & 1.7 & 1.9 & 2.2 & 2.6 & 3.1 \\
5 & 1.3 & 1.3 & 1.3 & 1.5 & 1.7 & 1.9 & 2.2 & 2.7 & 3.1 \\
10 & 1.3 & 1.2 & 1.3 & 1.5 & 1.6 & 1.8 & 2.0 & 2.4 & 2.8 \\
25 & 1.3 & 1.2 & 1.2 & 1.3 & 1.5 & 1.6 & 1.8 & 2.1 & 2.5 \\
\hline
\end{tabular}

\subsection{Asymptotic solutions}

For the case of the line contact problem the minimum or central film thickness could be solved analytically in some simplified situations, e.g. refs. $7-10$, which resulted in the well known asymptotic solutions. In the point contact situation the additional dimension makes an analytical solution of the problem, even in the restricted situations, complicated, if not impossible, and only a few asymptotic solutions for minimum or central film thickness are known to the authors.

Firstly, the rigid isoviscous asymptote is discussed, i.e. the equivalent of the Martin solution for the line contact problem. A straightforward dimensional analysis shows that this asymptote can be written as

$H_{\min }=C M^{-2}$

where $H_{\min }$ denotes the Moes dimensionless minimum film thickness parameter (see nomenclature). The remaining problem is to determine the value of the constant. In a more general sense, i.e. for elliptical contacts, this subject has been addressed by, among others, Kapitza [11] and also by Brewe et al. [12]. For a circular contact Kapitza's analysis gives $C=28.4$ whereas according to Brewe et al: $C=35.0$. The relatively large difference between these two values can be ascribed to the different types of analysis used. In particular, Kapitza's prediction is based on half Sommerfeld solutions, i.e. disregarding cavitation.

The authors solved this specific asymptotic problem numerically. For the circular contact $C=35.5$ was obtained, see ref. 13. This value was already reported by Lubrecht [2]. Comparing this result with the value presented by Brewe et al. it can be stated that this asymptotic solution is established rather accurately. Hence, it is justified to adopt the following formula for the rigid isoviscous asymptote for the circular contact:

$H_{\min }=35.5 M^{-2}$

In this asymptotic situation in which elastic deformation is absent the minimum film thickness equals 0.75 times the central film thickness. Consequently, the dimensionless central film thickness is given by

$H_{\text {cen }}=47.3 M^{-2}$

The second asymptotic situation considered is referred to as elastic-isoviscous. This asymptote is of particular interest for situations in which at least one of the surfaces has a small stiffness, e.g. in the case of seals. In terms of the Moes dimensionless parameters these situations are characterized by $L=0$ and large $M$.

Contrary to the situation described by eqns. (2) and (3) now the elastic deformation is of great importance for the fluid film formation. All solutions for large $M$ and $L \approx 0$ 
clearly show this elastic deformation for example in the large region of nearly unitorm film thickness and the formation of the side lobes, see e.g. Fig. 7. Consequently, to describe this asymptotic situation in fact both a formula for the minimum as well ats a formula for the central film thickness are required.

A solution for long elliptical contacts with the major axis perpendicular to the direction of rolling was derived by Moes [10]

$H_{\min }=2.34 M^{-2 / 15}$

However, because the analysis leading to this equation neglects the side flow, it will probably overestimate the film thickness when applied to the circular contact situation. For that situation Moes proposed

$H_{\min }=1.31 M^{-2 / 15}$

By means of the analytical solution of Reynolds' equation on the centerline of the contact under the assumption of a circular symmetric pressure profile and the elastic deformation given by Hertz [14], the author derived the following expression for the central film thickness, see ref. 13:

$H_{\text {cex }}=0.97 M^{-2 / 15}$

Through a comparison of the predictions of this equation with numerically calculated values Lubrecht [2] showed that the predicted slope of $-2 / 15$ was fairly accurate but that the constant was too small.

The three formulas (4) to (6) are based entirely on analytical arguments. Alternatively Hamrock and Dowson [15] curve-fitted the results of some numerically calculated solutions and presented formulas for both the central and the minimum film thickness in the elastic isoviscous situation. In terms of the Moes dimensionless parameters their equation for the minimum film thickness reads

$H_{\min }=2.035 M^{-0.21}$

whereas their equation for dimensionless central film thickness reads

$H_{\text {cen }}=5.29 M^{-0.22}$

Note that there is only a slight difference in the way in which the minimum film thickness and the central film thickness depend on the load $(M)$. This does not seem to agree with results of numerical calculations presented by Kweh et al. [3] and Lubrecht [2] who found that the minimum film thickness decreases much faster with increasing $M$ than the central film thickness does. This subject will be addressed in more detail in section 3.2 .

For the third asymptotic situation for the line contact problem, i.e. the Grubin solution, the author derived the following equation:

$H_{c e n}=1.02 M^{-1 / 12} L^{3 / 4}$

This formula was obtained solving Reynolds' equation on the centerline of the contact assuming a circular symmetric pressure profile and the elastic deformation given by Hertz [14], see ref. 13. Comparison of the predictions of this formula with calculational results showed that it was relatively inaccurate.

\subsection{Film thickness diagrams}

Figure 8 displays the presently calculated values of $H_{\min }$ as a function of $M$ and $L$. The most extreme situation represented in this diagram is $M=1000$ and $L=25$. Alternatively this case is described by $\bar{\alpha}=91.09$ and $\lambda=1.10 \times 10^{-3}$. Values of the 
Hamrock and Dowson parameters describing these conditions are: $W=9.46 \times 10^{-6}$, $U=7.81 \times 10^{-10}$, and $G=4729$. With $\alpha=1.7 \times 10^{-8}$ the maximum Hertzian pressure for this load situation is $5.35 \mathrm{GPa}$. The reader is reminded that solutions of the smooth surface circular contact problem for such extreme loads in itself are of little practical relevance for many reasons. Among things, the viscosities are unrealistically high. Moreover, at such high pressures the lubricant will most probably behave as a solid instead of a fluid. Furthermore, in the case of steel surfaces beyond $3.3 \mathrm{GPa}$ gross plastic deformation of the raceways will occur which is also not included in the model. However, the fact that the smooth surface problem can be solved for such high loads, (extremely small coefficient values in the entire contact region) gives confidence that the algorithm will also enable the study of the aforementioned more complex EHL situations (extremely small coefficients locally).

The drawn line in Fig. 8 represents the rigid isoviscous asymptote, i.e. eqn. (2). In addition the elastic isoviscous asymptote according to Hamrock and Dowson [15] (eqn. (7)) is plotted. The figure shows that this latter asymptotic solution compares favourably with the numerically calculated values although for large $M(=1000)$ the calculated value falls somewhat below the prediction of eqn. (7). Also for $L>0$ and large $M$ the dimensionless minimum film thickness shows a tendency to decrease with a larger slope than eqn. (7) predicts. This probably is a numerical effect caused by the fact that for high values of $M$ the sidelobes become very small, see Fig. 7 or ref. 1, Fig. 9.13. As a result at least locally a large nodal density is required to obtain an accurate estimate of its value and location. This only applies to the minimum film thickness. An accurate estimate of the central film thickness does not requirc such large nodal densities.

This observation probably explains why the values of $H_{\min }$ for $M \geq 100$ and small $L$ presented by Lubrecht [2] are much smaller than those obtained from the present calculations for the same conditions whereas the value of the central film thickness is about the same. This need for a large local nodal density to accurately represent the sidelobes in these situations also becomes evident from comparing the film thickness contour plots presented in ref. 2, pp. 132-135 with those for the same conditions presented in Fig. 7 and ref. 1, Fig. 9.13.
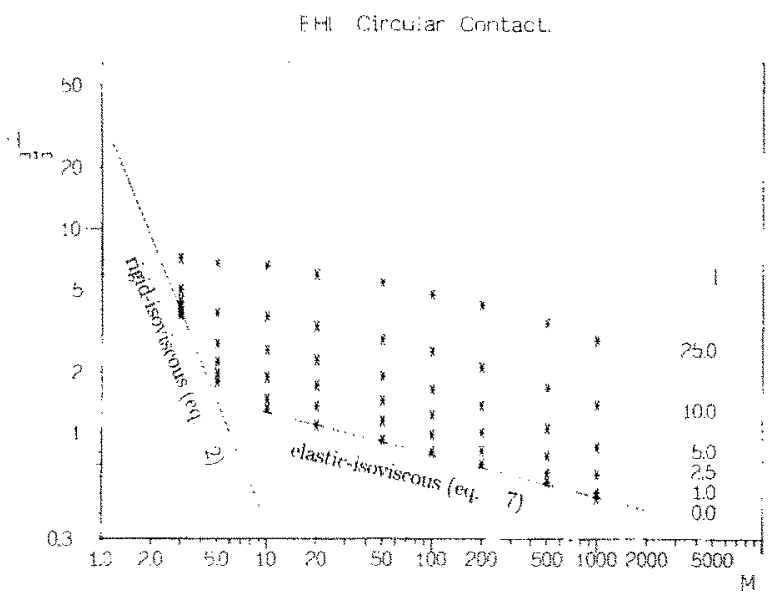

Fig. 8. Calculated values of the dimensionless minimum film thickness parameter $H_{\min }\left({ }^{*}\right)$ as a function of $M$ and $L$. The drawn lines give the predictions of eqns. (2) and (7) respectively. 
Figure 9 shows the presently calculated values of the dimensionless central film thickness, $H_{\text {cen }}$, as a function of $M$ and $L$. The drawn lines indicate the predictions of this film thickness according to a formula presented in Section 3.3. Clearly the calculational results and the predictions of this formula match quite accurately.

Finally, Fig. 10 presents the calculated values of $H_{\text {av, }}$ the dimensionless average film thickness, as a function of $M$ and $L$, where the average is taken over the Hertzian contact region $\left(X^{2}+Y^{2} \leq 1\right)$.

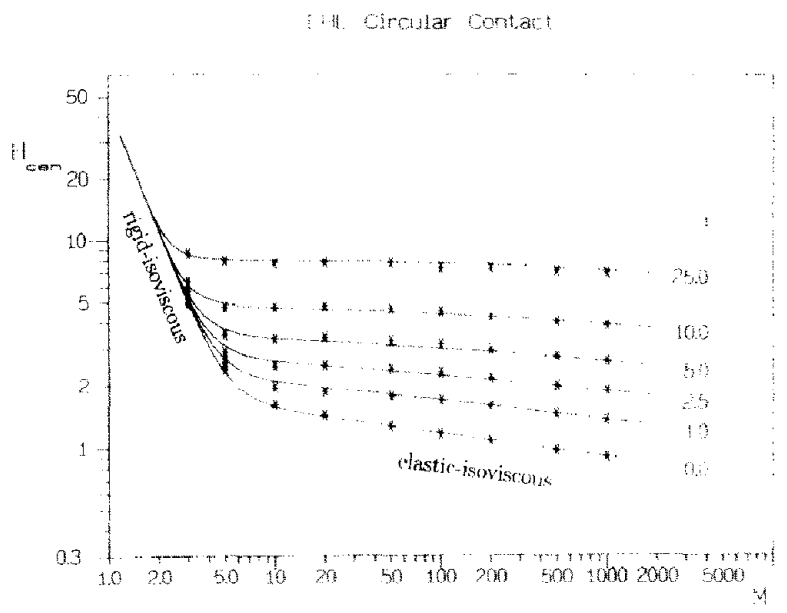

Fig. 9. Calculated values of the dimensionless central film thickness parameter $H_{\text {cen }}{ }^{*}$ ) as a function of $M$ and $L$. The drawn lines give the predictions of eqns. (12) presented in section 3.3 .
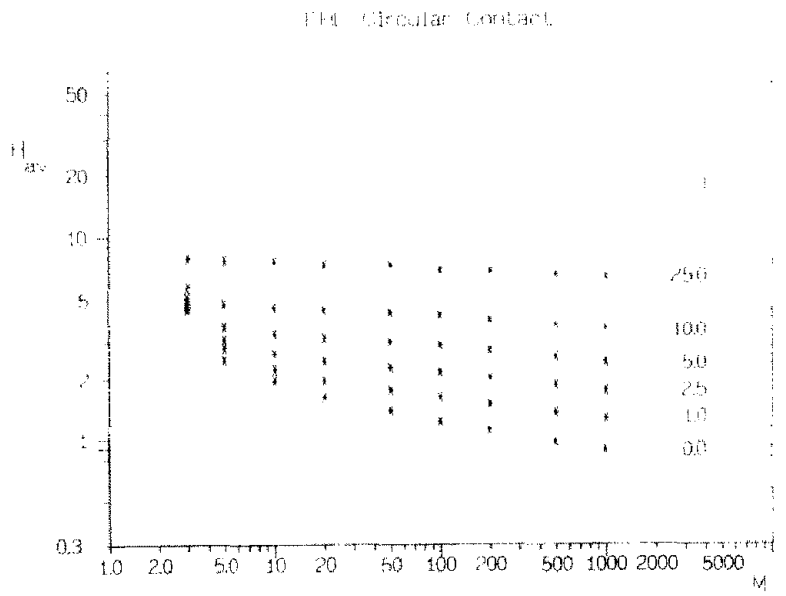

Fig. 10. Calculated values of the dimensionless average film thickness parameter $H_{3 v}\left(^{*}\right)$ as a function of $M$ and $L$. 


\subsection{Film thickness formula}

From the calculational results the following asymptote for the central film thickness in the elastic isoviscous regime was derived:

$H_{\text {cen }}=1.96 M^{-1 / 9}$

The predictions of this formula accurately approach the calculated values for large $M$ and $L=0$. Note that the slope $-1 / 9$ is less steep than the slope of the Hamrock and Dowson minimum film thickness asymptote (eqn. (7)) for these conditions. This clearly shows that the minimum film thickness decreases much faster with load than the central film thickness does.

For the same situation (large $M$ ) but with $L>0$ the following equation quite accurately predicts the central film thickness:

$H_{\text {cen }}=1.70 M^{-1 / 9} L^{3 / 4}$

Using three parameters $r, s$ and $t$ to take care of a smooth transition in the intermediate region the equations (3), (10) and (11) can be merged into one expression that predicts the dimensionless central film thickness over the entire parameter range. This formula was developed by Moes [10] and reads

$H_{\text {cen }}=\left\{\left[\left(1.70 M^{-1 / 9} L^{3 / 4} t\right)^{r}+\left(1.96 M^{-1 / /}\right)^{r}\right]^{s / r}+\left(47.3 M^{-2}\right)^{s}\right\}^{1 / s}$

where $r, s$ and $t$ are given by

$r=\exp [1-6 /(L+8)]$

$s=12-10 \exp \left(M^{-2}\right)$

and

$t=1-\exp \left(-0.9 \frac{M^{1 / 6}}{L^{1 / 6}}\right)$

At first sight this equation may seem complex; however, a closer look shows that the structure is quite straightforward and allows easy programming on a pocket or personal computer.

The values for the dimensionless central film thickness predicted by eqn. (12) are indicated by the drawn lines in Fig. 9. When compared with the calculated values it shows that this formula accurately predicts the central film thickness in the entire parameter regime considered in the present study.

\section{Conclusions}

The multilevel algorithm developed for the solution of the EHL circular contact problem has been applied to the situation where the surfaces are perfectly smooth. The variation of the pressure profile and particularly the variation of the film thickness was studied over a wide range of parameter values. This parametric study has resulted in an accurate formula for the prediction of the central film thickness.

Although the subject was discussed only briefly, the pressure profiles presented in this paper show that, as was found earlier for the line contact (see ref. 6), the (three-dimensional equivalent of) the pressure spike, only occurs in part of the parameter regime. In addition, for a given $M$ it develops gradually from a small ridge into a real "spike" when the dependence of the viscosity on the pressure increases. 


\section{Acknowledgments}

The authors would like to thank Professor A. Brandt of the Weizmann Institute of Science, Rehovot, Israel and Dr. A. A. Lubrecht of the SKF Engineering \& Research Centre, Nieuwegein, Netherlands, for the many helpful discussions with respect $t o$ the development of the algorithm. Furthermore, the authors wish to thank H. Moes from the tribology group of the University of Twente, Enschede, Netherlands for curvefitting the central film thickness results into a single formula. This research was sponsored by the SKF Engineering \& Research Centre. The photographs presented in this paper were made by TTF, Enschede, Netherlands.

\section{Nomenclature}

$a$ half-width Hertzian contact region, $\left.a=\left(\left(3 w R_{x}\right) / 2 E^{\prime}\right)\right)^{1 / 3}$

$E \quad$ elasticity modulus (Young's modulus)

$E^{\prime} \quad$ reduced modulus of elasticity, $2 / E^{\prime}=\left(1-\nu_{1}^{2}\right) / E_{1}+\left(1-\nu_{2}^{2}\right) / E_{2}$

$G \quad$ material parameter, $G=\alpha E^{\prime}$

$h \quad$ film thickness

$H$ dimensionless film thickness, $H=(h R) / a^{2}$

$H_{00} \quad$ integration constant in dimensionless film thickness equation

$H_{\min }$ dimensionless minimum film thickness parameter, $H_{\min }=\left(h_{\min } / R_{x}\right)(2 U)^{-1,2}$

$H_{\text {cen }}$ dimensionless central film thickness parameter (Moes), $H_{\text {cen }}=\left(h_{\text {cen }} / R_{x}\right)$ $(2 U)^{-1 / 2}$

$H_{\mathrm{av}}$ dimensionless average film thickness parameter (Moes), $H_{\mathrm{av}}=\left(h_{\mathrm{av}} / R_{x}\right)$ $(2 U)^{-1 / 2}$

$L \quad$ dimensionless material parameter (Moes), $L=G(2 U)^{1 / 4}$

$M \quad$ dimensionless load parameter (Moes), $M=W(2 U)^{-3 / 4}$

p pressure

$p_{\mathrm{h}} \quad$ maximum Hertzian pressure, $p_{\mathrm{h}}=(3 F) /\left(2 \pi a^{2}\right)$

$P \quad$ dimensionless pressure, $P=p / p_{\mathrm{h}}$

$R \quad$ reduced radius of curvature, $R^{-1}=R_{1}{ }^{\cdots 1}+R_{2}{ }^{\cdots 1}$

$u_{\mathrm{s}} \quad$ sum velocity, $u_{\mathrm{s}}=u_{1}+u_{2}$

$U$ dimensionless speed parameter, $2 U=\left(\eta_{0} u_{\mathrm{s}}\right) /\left(E^{\prime} R\right)$

$F \quad$ load per unit width

$W \quad$ dimensionless load, $W=F /\left(E^{\prime} R\right)$

$x \quad$ coordinate

$X$ dimensionless coordinate, $X=x / a$

$y \quad$ coordinate

$Y$ dimcnsionless coordinate, $Y=y / a$

$\alpha \quad$ pressure viscosity index

$\bar{\alpha} \quad$ dimensionless parameter, $\bar{\alpha}=\alpha p_{\mathrm{h}}$

dimensionless vclocity parameter, $\lambda=6\left(\eta_{0} u_{\mathrm{s}} R^{2}\right) /\left(b^{3} p_{\mathrm{h}}\right)$

Poisson's ratio

viscosity

$\eta_{0} \quad$ viscosity at atmospheric pressure

$\bar{\eta}$

$\rho$ density

$\rho_{0} \quad$ density at atmospheric pressure

$\bar{\rho} \quad$ dimensionless density, $\bar{\rho}=\rho / \rho_{0}$ 


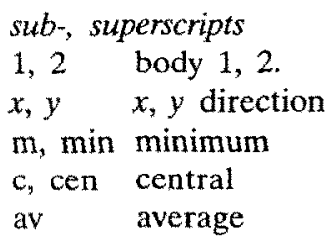

\section{References}

1 C. H. Venner, Multilevel solution of the EHL line and point contact problem, $\mathrm{Ph} . \mathrm{D}$. Thesis, Univ, of Twente, Enschede, The Netherlands, 1991.

2 A. A. Lubrecht, The numerical solution of the elastohydrodynamically lubricated line- and point contact problem using multigrid techniques, Ph.D. Thesis, Univ. of Twente, Enschede, 1987; ISBN 90-9001583-3.

3 C. C. Kweh, H. P. Evans and R. W. Snidle, Elastohydrodynamic lubrication of heavily loaded circular contacts, Proc. Inst. Mech. Eng., 203 (1989) 133-148.

4 C. J. A. Roelands, Correlational aspects of the viscosity-temperature-pressure relationship of lubricating oils, Ph.D. Thesis, Technische Hogeschool Delft, The Netherlands, 1966 (V.R.B., Groningen, The Netherlands.)

5 D. Dowson and G. R. Higginson, Elasto-hydrodynamic lubrication, The fundamentals of roller and gear lubrication, Pergamon, Oxford, 1966.

6 C. H. Venner and W. E. ten Napel, Numerical calculations of the pressure spike in EHL, Proc. EUROTRIB 89, Vol. 2, 1990, pp. 196-201.

7 H. M. Martin, Lubrication of gear teeth, Engineering (London), 102 (1976) 199.

8 A. M. Ertel, Hydrodynamic lubrication based on new principles, Akad. Nauk SSSR Prikadnaya Math. Mekh., 3 (2) (1939) 41-52.

9 A. N. Grubin and I. E. Vinogradova, Investigation of the contact of machine components, $\mathrm{Kh}, \mathrm{F}$. Ketova (ed.), Central Scientific Research Institute for Technology and Mechanical Engineering (Moscow), Book No. 30, (DSIR translation No. 337) (1949).

10 H. Moes, internal memo on asymptotical film thickness calculations, Univ. of Twente, Enschede, The Netherlands, 1986.

11 P. L. Kapitza, Hydrodynamic theory of lubrication during rolling, Zh. Tekh. Fiz., 25 (1955) 747.

12 D. Brewe, B. J. Hamrock and C. M. Taylor, Effect of geometry on hydrodynamic film thickness, ASME JOT, 101 (1979) 231-239.

13 C. H. Venner, M.Sc. Thesis, Univ. of Twente, Enschede, The Netherlands, 1987 (in Dutch).

$14 \mathrm{H}$. Hertz, Über die Berübrung fester elastischer Körper, J. Reine Angew. Math, 92 (1881) 156-171.

15 B. J. Hamrock and D. Dowson, Elastohydrodynamic lubrication of elliptical contacts for materials of low elastic modulus 1 - Fully flooded conjunction, ASME JOT, 100 (1978) 236-245. 